Article

\title{
Simulation of a Narrowband Power Line Communications System over Medium Voltage
}

\author{
Nikolaos Chiotellis ${ }^{1, *,+}$ and Panayotis G. Cottis ${ }^{2, t}$ \\ 1 Department of Electrical Engineering and Computer Science, University of Michigan, Ann Arbor, \\ MI 48109-2122, USA \\ 2 School of Electrical and Computer Engineering, National Technical University of Athens, Zografou 15780, \\ Greece; pcottis@central.ntua.gr \\ * Correspondence: nchiot@umich.edu; Tel.: +1-734-834-9536 \\ + These authors contributed equally to this work.
}

Academic Editor: Hung-Yu Wang

Received: 28 September 2015; Accepted: 14 March 2016; Published: 23 March 2016

\begin{abstract}
Narrowband Power Line Communications (NB-PLCs) are investigated as an alternative option for transferring low rate smart grid (SG) data via Medium Voltage (MV) power lines. In this framework, two variants of orthogonal frequency division multiplexing are examined, namely Filtered-OFDM (F-OFDM) and Wavelet-OFDM (W-OFDM), in an attempt to determine which of them is capable of transmitting low rate SG data to greater distances over non-branched MV power lines. The reach of NB-PLC signals via MV power lines is estimated, taking into account the transfer function of the relevant PLC channels and noise mechanisms as well as the specific features of the two modulation options under consideration. Simulations show that NB-PLC transmission constitutes a technically feasible and economically affordable option for exchanging low rate data with remote SG nodes dispersed over the MV grid. Moreover, simulations show that F-OFDM allows low rate data transmission to considerably greater distances compared to W-OFDM.
\end{abstract}

Keywords: Filtered-OFDM; medium voltage; Nakagami; power line communications; smart grid; Wavelet-OFDM

\section{Introduction}

The Smart Grid (SG) is a power network which can intelligently integrate/incorporate the actions of generators, distributors and consumers of energy. Employing information and communication technologies (ICT), the SG aims at delivering electrical power in a sustainable, economic and secure way [1]. In addition to enhancing the efficiency, reliability and safety of power delivery, the SG will also: (i) increase the penetration and enhance the smooth integration of distributed renewable electric power generation, (ii) implement the practically real-time participation of energy producers and consumers in the energy market, and (iii) push forward the use of electrical vehicles. All of these enhanced applications and services necessitate ICT solutions customized to transmission and distribution networks [2,3].

The benefits expected by Distribution System Operators (DSOs) and end users from the SG are operational, economic, environmental and security specific. As a result, many DSOs intend to deploy last mile communication infrastructure to monitor crucial network operations and exchange information with critical nodes of the transmission and the distribution grid (e.g., generators, substations, large consumers). Specifically, DSOs have the options of using: (i) Power Line Communications (PLC), to move digital information through the grid via the very power lines used for the transmission and distribution of electric power; or (ii) wireless communication technologies, to communicate with substations and implement services primarily related to remote metering. 
The SG communications (SGC) architecture comprises two layers, the power system layer, responsible for the safety and reliability of the distribution grid, and the communications layer, intended for the exchange of information between nodes of the power grid. Broadband PLC (BB-PLC), also referred to as Broadband over Power Lines (BPL), is a technology appropriate for last mile access. As verified by in situ measurements from various pilot PLC installations, BB-PLC enables broadband transmission in the 1-100 $\mathrm{MHz}$ frequency range at raw data rates exceeding $200 \mathrm{Mbps}$ [4]. Though both the low voltage (LV) and the 1-33 kV MV grid can be employed for BB-PLC transmission, the latter is subjected to less adverse transmission conditions, making feasible the deployment of BB-PLC networks over the MV grid at acceptable QoS levels. Combined with the recently released IEEE P1901 standard [5] and the ubiquitous presence of the MV grid, BB-PLC technology seems attractive for SG applications destined for the MV grid [6].

Narrowband PLC (NB-PLC) refers to low data rate communications utilizing the frequency band either below $148.5 \mathrm{kHz}$ in Europe (following the CENELEC standard: 3-148.5 kHz) or below $450 \mathrm{kHz}$ in the USA and several Asian countries. NB-PLC technology utilizes the power grid to implement low rate network services related to switching and monitoring. However, both PLC options face a harsh transmission environment characterized by a time-varying transfer function and, sometimes, non-white additive noise of high power spectral density (PSD) [7]. The propagation environment faced by the two PLC options varies. In particular, the attenuation in the relatively narrow NB-PLC frequency range is quite low and exhibits smaller variations. On the other hand, in the significantly broader BB-PLC frequency range, the attenuation exhibits a non-monotonic behavior in a much broader range of values. In both cases, signal transmission is impeded, necessitating the use of orthogonal frequency division multiplexing (OFDM) techniques. On the other hand, though the additive white Gaussian noise (AWGN) assumption is valid for the noise encountered in BB-PLC, it is not valid for NB-PLC, where the relevant noise exhibits a non-white and non-Gaussian behavior, a feature that must be taken into account in NB-PLC transmission analysis.

BB-PLC operates over a significantly broader frequency range, thus being capable of supporting high data rate SG applications. As NB-PLC systems operate in a low and relatively narrow frequency range, they seem suitable for SG applications operating at low data rates (up to $144 \mathrm{kbps}$ ). In particular, NB-PLC transmission via MV power lines seems to be a promising SGC option as the MV grid runs through longer distances and serves remote and isolated areas, where the use of other SGC options might not be affordable. This work investigates how NB-PLC employing non-branched MV lines can increase the reach of low rate SG data in remote and/or isolated areas. To that end, multipath propagation is ignored. The transmission environment considered takes into account the transfer function of the NB-PLC channel encountered in MV power lines and its noise characteristics. Since PLC transmission employs primarily OFDM, this article investigates the performance of two such modulation schemes, namely filtered-OFDM (F-OFDM) and wavelet-OFDM (W-OFDM). These modulation schemes are only used indicatively to demonstrate the fact that NB-PLC is a viable SGC option via MV grids, in addition to its use over LV grids. Error correction techniques, such as low-density parity-check code or convolution code, which are well established in the usual practice, may also significantly extend the range of NB-PLC systems. Additionally, adaptive modulation or power allocation techniques (e.g., using a water filling algorithm) may enhance the reach of NB-PLC signals through the MV grid.

The simulations performed by the authors have shown that low rate data can be transmitted via non-branched MV power lines to distances up to $2.5 \mathrm{~km}$ compared to significantly lower distances-of the order of 500-800 m-attainable employing BB-PLC. The simulations have also shown that F-OFDM modulated signals are capable of reaching significantly greater distances than the W-OFDM ones. When a robust modulation scheme is used (e.g., 2-QAM), a bit error ratio (BER) in the order of $10^{-4}$ is attainable.

The rest of the article is organized as follows. In Section 2, NB-PLC transmission in multi-conductor overhead MV power lines is outlined. In Section 3, the corresponding noise model is 
discussed considering the primary noise mechanisms and sources encountered in MV power lines. In Section 4, the two OFDM options under investigation with regard to NB-PLC transmission are briefly discussed. Section 5 presents the obtained simulation results that determine the maximum reach of NB-PLC signals before having to be repeated. Finally, Section 6 concludes the article.

\section{PLC Transmission via Overhead MV Power Lines}

Signal transmission via MV power lines differs considerably from signal transmission via twisted-pair, coaxial, or fiber-optic cables due to specific characteristics related to the topology and the physical properties of power lines [8]. In typical overhead MV installations, power lines hang above lossy ground at a height ranging from 8 to $10 \mathrm{~m}$. They normally consist of the three phases, separated by 0.3 to $1 \mathrm{~m}$, and a ground return. To determine the transfer function of multi-conductor transmission lines (MTL) employed for PLC transmission, the well-known two-conductor analysis is extended employing appropriate matrix analysis [8].

\section{Two-Conductor Transmission Lines}

The line voltage at distance $L$ from the injection point is given by

$$
V(L)=H(f, L) V(0),
$$

where

$$
H(f, L)=e^{-\gamma(f) L}=e^{-\alpha(f) L} e^{-j \beta(f) L}
$$

is the transfer function of the transmission line at this distance. The frequency response of a matched two-conductor power line used for PLC transmission is determined via the complex propagation constant

$$
\gamma(f)=\alpha(f)+j \beta(f) .
$$

Among the various approaches proposed to determine $\gamma(f)$, taking into account the topology and the electromagnetic parameters of the power grid, the current article follows [9] as it applies to the whole PLC frequency range and can easily be extended to MTL channels.

\section{Multi-Conductor Transmission Lines}

An MTL consisting of $n+1$ lines parallel to the $z$-axis supports $n$ forward and $n$ backward traveling waves propagating with distinct propagation constants. These waves are jointly described by a coupled set of $2 n$, first-order, partial differential equations relating the line voltages $V_{i}(z, t)$ and the line currents $I_{i}(z, t), i=1,2, \ldots, n$. Each pair of forward and backward traveling waves constitutes a mode [7,8]. An MTL consisting of four conductors and a ground return supports four modes, whereas a three-conductor MTL with ground return supports three modes. The modal wire to ground voltages $V_{m}(z)=\left[V_{1}^{m}(z) V_{2}^{m}(z) \ldots V_{n}^{m}(z)\right]^{T}$ and modal wire currents $\boldsymbol{I}_{m}(z)=\left[\begin{array}{llll}I_{1}^{m}(z) & I_{2}^{m}(z) & \ldots & I_{n}^{m}(z)\end{array}\right]^{T}$ are related to the line voltages $\boldsymbol{V}(z)=\left[\begin{array}{llll}V_{1}(z) & V_{2}(z) & \ldots & V_{n}(z)\end{array}\right]^{T}$ and currents $I(z)=\left[\begin{array}{llll}I_{1}(z) & I_{2}(z) & \ldots & I_{n}(z)\end{array}\right]^{T}$ via the similarity transformation

$$
\begin{gathered}
\boldsymbol{V}(z)=\boldsymbol{T}_{V} \boldsymbol{V}_{m}(z), \\
\boldsymbol{I}(z)=\boldsymbol{T}_{I} \boldsymbol{I}_{m}(z),
\end{gathered}
$$

where $\boldsymbol{T}_{V}$ and $\boldsymbol{T}_{I}$ are $n \times n$ matrices that depend on frequency, the physical properties of the power lines and the lossy earth, and the geometry of the MTL configuration. Equations (4a) and (4b) relate the line voltages and currents with the corresponding modal quantities. Under specific assumptions [8], the three modes propagating along three-wire overhead MV power lines may be decoupled, giving rise to three independent transmission channels which can simultaneously transfer PLC signals. The common (or ground) mode is characterized by a comparatively higher attenuation 
and propagates via all the phases (wires) returning via the ground. The differential (or aerial) modes, propagating and returning via the wires, exhibit a lower attenuation compared to the common mode [7]. In PLC transmission, depending on the way the signal is coupled onto the power lines, either wire-to-wire (WTW) or wire-to-ground (WTG) injection is possible. Under WTW injection, differential modes are mostly excited. Under WTG injection with coupling to the middle phase, both the common mode and the differential modes are excited. Under certain conditions, which are practically satisfied for frequencies up to $100 \mathrm{MHz}$, the PLC modes supported by MV power lines are orthogonal [10].

\section{Noise in PLC Transmission}

\subsection{Noise Considerations in PLC Channels}

In general, PLC channels are affected by strong, time-varying, non-white, non-Gaussian noise [8]. Measurements of the background noise level in an MV power line test bed [11,12] have shown that, in the frequency range $1-20 \mathrm{MHz}$, the noise levels observed are approximately 20-30 $\mathrm{dB}$ higher than the noise levels observed in in-house LV lines. In addition, the noise in power lines exhibits periodicity synchronous to the main voltage due to the relevant noise generated by the various electric devices connected. This periodic noise cannot be ignored since, due to the relatively narrow operational bandwidth exploited by NB-PLC transmission, the symbol duration and, consequently, the packet length, tend to be long. As the noise at higher frequencies is attenuated more, the noise affecting PLC transmission exhibits a higher PSD at lower frequencies. Furthermore, many noise sources are active mainly in the lower PLC frequency range. In conclusion, the noise PSD affecting NB-PLC transmission in the $3-500 \mathrm{kHz}$ band is not Gaussian and exhibits an approximately exponential decay with frequency.

The noise encountered in MV power lines is a mix of periodic impulse noise, background noise and narrowband noise [12]. The background noise is primarily dependent on the weather conditions, the terrain profile and conditions, etc. [7]. Moreover, as PLC systems share frequency bands with primary wireless services, the latter cause interference to PLC channels on a local basis, which is perceived as narrowband noise [4]. Finally, background noise in MV lines is caused by corona discharges, especially under humid and heavy weather conditions [13]. When referring to narrow frequency ranges such as the NB-PLC frequency range under consideration, the corona noise is perceived as white noise.

\subsection{Noise Characteristics Verified from in situ Measurements}

In situ measurements concerning the noise involved in MV PLC transmission have resulted in the following conclusions [12]:

- Every substation gives rise to a unique noise distribution.

- The transformers that electrically separate the MV grid from the HV and LV ones prevent the transition either of noise or of harmonic distortion products.

- The noise PSD in MV power lines is found to be higher than in LV ones by 20-30 dB.

- The maximum and minimum noise PSD levels may differ by up to $25 \mathrm{~dB}$, indicating that corona noise from HV power lines is induced into the MV grid through the air.

- The noise PSD is affected by load fluctuations, especially in the NB-PLC frequency range.

- The noise levels observed in phase-to-phase (WTW) coupling does not usually exceed the ones observed in the relevant two single-phase-to-ground (WTG) couplings.

The two primary properties taken into account to model noise in NB-PLC systems are:

- The noise cumulative density function $(\mathrm{CDF})$ can be better approximated by the Nakagami distribution and not by the Gaussian distribution as commonly assumed in the standard analysis and design of communication systems. 
- The mean PSD of the total noise encountered in NB-PLC systems can be modeled by an exponentially decreasing function of frequency, that is

$$
\operatorname{PSD}(f)=A e^{B f}
$$

where $A$ and $B$ are quantities depending on the parameters of the substation [12,14].

\section{OFDM in PLC Transmission}

The IEEE P1901 standard for PLC systems proposes two filter bank multicarrier (FBMC) techniques, namely F-OFDM and W-OFDM $[5,15]$. Here, these two modulation options are studied for NB-PLC transmission via non-branched MV power lines in the attempt to determine the maximum reach of NB-PLC signal transmission.

\subsection{Filtered-OFDM}

As in conventional OFDM, inputs to an F-OFDM system are signals of the form

$$
s_{k}(t)=\sum_{n} s_{k}[n] \delta(t-n T),
$$

where $s_{k}[n]$ are the subcarrier data symbols, $k$ is the subcarrier index and $T$ is the symbol duration. In general, the difference between OFDM and FBMC lies in the choice of the impulse responses of the shaping filters $p_{T}(t)$ and $p_{R}(t)$ used at the transmitter and the receiver, respectively. In OFDM, $p_{T}(t)$ is a rectangular pulse of duration equal to $T$, whereas $p_{R}(t)$ is also a rectangular pulse but of smaller duration $T_{F T T}<T$, where $T_{F T T}=1 / B$ and $B$ is the subcarrier frequency spacing. In FBMC systems designed for maximum spectral efficiency, the duration of both shaping pulses is chosen equal to $T_{F T T}$. Moreover, the durations of $p_{T}(t)$ and $p_{R}(t)$ are longer than the symbol duration $T$ (usually an integer multiple of $T$ ), causing the overlapping of successive data symbols [16].

Under distortionless transmission, a simple choice for $p_{T}(t)$ and $p_{R}(t)$ would be a pair of rectangular pulses both of duration $\tau$. When channel distortion exists, the transmitted symbols undergo a transient state before reaching the steady state. To alleviate the transient phenomenon, the duration of $p_{T}(t)$ is extended in time by a time period greater than the duration of the channel impulse response by adding a cyclic prefix $(\mathrm{CP})$ to every OFDM symbol. At the receiver, $p_{R}(t)$ is aligned in time with the transmitted symbol after the latter has reached its steady state.

In F-OFDM, the rectangular shaping pulse is replaced by a pulse (window function) exhibiting a smoother transition at the edges. A $p_{T}(t)$ option often used in practical F-OFDM transmission is the raised cosine pulse

$$
p_{T}(t)=\Pi\left(\frac{t-T / 2}{T}\right) \otimes h(t),
$$

where $\otimes$ denotes convolution, and

$$
\begin{gathered}
\Pi\left(\frac{t}{T}\right)= \begin{cases}1, & |t| \leq T / 2 \\
0, & \text { otherwise }\end{cases} \\
h(t)=\frac{\pi}{2 T_{0}} \sin \left(\frac{\pi t}{T_{0}}\right) \Pi\left(\frac{t-T_{0} / 2}{T_{0}}\right),
\end{gathered}
$$

and $T_{0}$ is the overlapping period of adjacent F-OFDM symbols, referred to as the roll-off period.

\subsection{Wavelet-OFDM}

Wavelets are zero mean waveforms of fixed duration. The difference between wavelets and sinusoids employed in Fourier analysis is that wavelets are usually irregular and asymmetric. The wavelet transform decomposes the signals into shifted and scaled versions of appropriate wavelets. 
The advantage of the wavelet transform over other transforms is that it is discrete both in time and in scale. The wavelet decomposition is implemented employing appropriate low-pass (LPF) and high-pass (HPF) filters, followed by a down-sampler to enhance the spectral efficiency of the wavelet transform. According to the above procedure, the wavelet transform decomposes a signal into its approximation coefficients (output of the LPF) and its detail coefficients (output of the HPF). The inverse procedure is performed employing the inverse wavelet transform. In W-OFDM, the discrete wavelet transform (DWT) and the inverse DWT (IDWT) replace the FFT and IFFT transforms used at the transmitter and the receiver in conventional OFDM as depicted by the block diagram of W-OFDM transmission given in Figure $1[17,18]$.

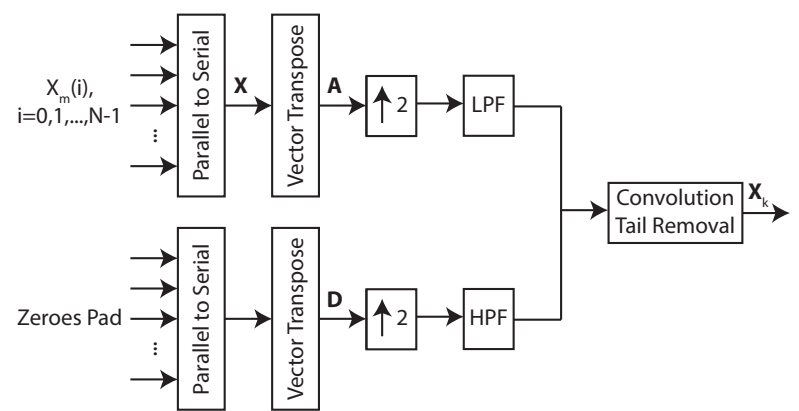

(a)

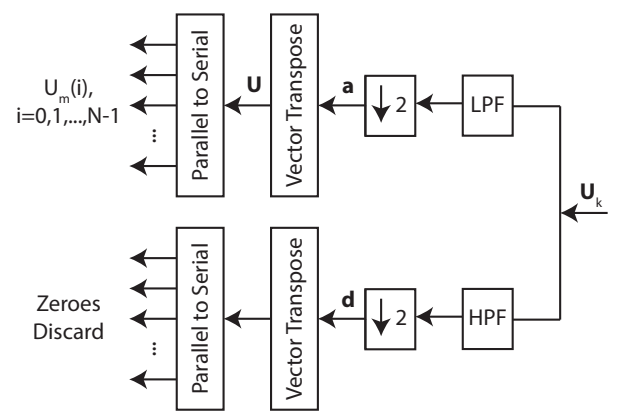

(b)

Figure 1. Wavelet-OFDM transmission [19]. (a) IDWT for transmission; (b) DWT for reception.

In Figure 1a, the transmitter uses a digital modulator which maps the input bits onto OFDM symbols $\mathbf{X}_{\mathbf{m}}$, forming $N$ parallel data streams $X_{m}(i), 0 \leq i \leq N-1$. Each stream $X_{m}(i)$ is converted to a serial stream forming a vector $\mathbf{X}$ which will be transposed into the approximation coefficients $\mathbf{A}$. To implement IDWT at the transmitter, the signal is up-sampled by two and then filtered by an LPF. This operation retains the primarily important lower part of the signal spectrum. Moreover, a zero padding signal is fed to an HPF. To produce the orthogonal wavelets required for the implementation of the wanted IDWT-DWT pair, the LPF and HPF filters must be quadrature mirror filters. The lengths of $\mathbf{A}$ and $\mathbf{D}$ depend on the number of OFDM subcarriers, $N$, and on the filters producing the wavelets selected [19]. The DWT procedure is implemented at the receiver as depicted in Figure 1b. The received data, $\mathbf{U}_{\mathbf{k}}$, are fed to the LPF and HPF employed at the receiver and are down-sampled by two. The a signal output from the LPF is then converted to a parallel stream, $U_{m}(i)$, which is fed to the demodulator. The $\mathbf{d}$ signal output from the HPF is discarded [19].

\section{Simulation Results and Discussion}

The simulations performed employing MATLAB aim at: (i) simulating the transmission environment of MV power lines in the NB-PLC frequency range and (ii) studying the relevant BER performance of F-OFDM and W-OFDM signals. The motivation is to derive BER curves in an attempt to determine the maximum distance to which NB-PLC signals can be reliably transmitted via non-branched MV power lines without having to be repeated. The values of the parameters used for the simulation of the two OFDM options examined are tabulated in Table 1.

\subsection{Evaluation of $\gamma(f)$ and $H(f)$}

The transfer function of the PLC channel examined is determined based on Section 2. The simulations for the real and imaginary part of the complex propagation constant $\gamma(f)$ give rise to the curves plotted in Figure 2a,b which are very close to the theoretical curves [9]. 
Table 1. Parameters of the simulated system.

\begin{tabular}{cccc}
\hline Number of Channels & 119 & Conductor Permeability & $\mu_{0}$ \\
\hline Symbol duration & $8.192 \mathrm{~ms}$ & Conductor permittivity & $2.3 \times 10^{3} \epsilon_{0}$ \\
\hline Modulation order & 2 or 4 & Conductor conductivity & $3.8 \times 10^{7} \mathrm{~S} / \mathrm{m}$ \\
\hline Number of symbols & 10,000 & Ground conductivity & $5 \times 10^{-3} \mathrm{~S} / \mathrm{m}$ \\
\hline Digital up-sampling factor & 16 & Ground permittivity & $13 \epsilon_{0}$ \\
\hline SNR & 7.5 to $20 \mathrm{~dB}$ & Ground permeability & $\mu_{0}$ \\
\hline Carrier frequency & $75.75 \mathrm{kHz}$ & Height of wires & $10 \mathrm{~m}$ \\
\hline Nyquist filter roll-off & 0.25 & Radius of wires & $2 \mathrm{~mm}$ \\
\hline Wavelet type & Haar & Distance between wires & $0.7 \mathrm{~m}$ \\
\hline W-OFDM overlap factor & 8 & Number of wires & 3 \\
\hline
\end{tabular}

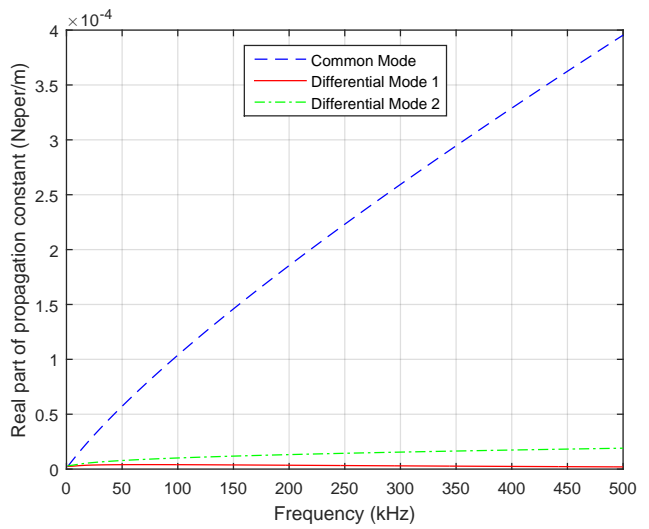

(a)



(b)

Figure 2. Frequency dependence of the complex propagation constant $\gamma(f)$ of MV-PLC channels. (a) $\alpha(f)=\operatorname{Re}(\gamma(f)) ;(\mathbf{b}) \beta(f)=\operatorname{Im}(\gamma(f))$.

In Figure 3, the transfer function amplitudes of the three modes supported by a three-wire MTL are plotted for an indicative distance of $2 \mathrm{~km}$.

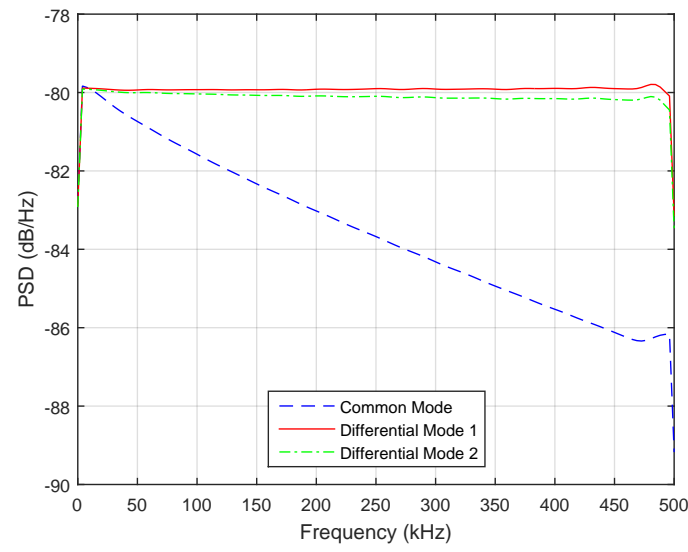

Figure 3. Amplitude of the transfer function for the three modes supported by a three-wire MTL at $2 \mathrm{~km}$ from the injection point. 


\subsection{The Additive Noise Model}

The noise superimposed on NB-PLC signals transmitted via MV power lines must exhibit the characteristics outlined in Section 3. In this context, noise samples, drawn from the Nakagami distribution with parameters $m=1$ and $\Omega=2$, are fitted in the spectral domain to an exponentially decreasing $\operatorname{PSD}(f)$. Figure 4a shows the distribution of noise samples generated by the simulations (simulated noise) together with the Nakagami distribution used to create the relevant noise samples (theoretical noise). It is deduced that filtering the noise in order to simulate an exponentially decreasing $\operatorname{PSD}(f)$ only slightly modifies the samples' distribution. Figure $4 \mathrm{~b}$ depicts the simulated noise $\operatorname{PSD}(f)$ in the frequency range of $0-500 \mathrm{KHz}$. It is readily verified that the simulated noise PSD possesses an exponentially decreasing mean value, at the same time preserving spectral randomness.



(a)

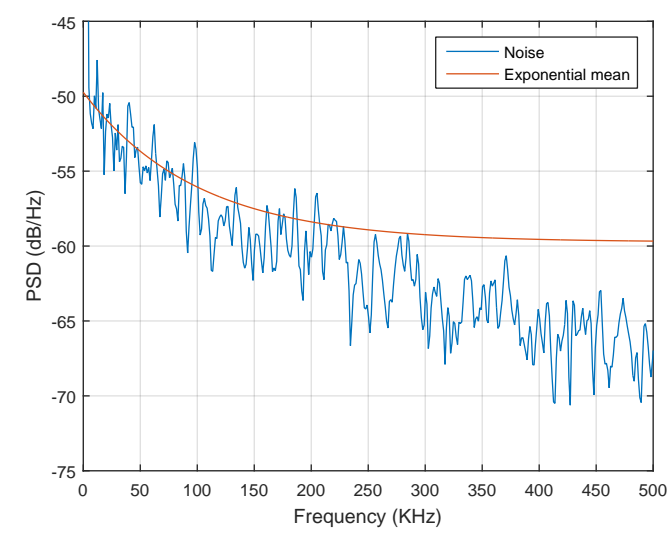

(b)

Figure 4. (a) CDF of the noise superimposed on NB-PLC signals transmitted via MV power lines; and (b) $\operatorname{PSD}(f)$ of simulated colored Nakagami noise.

\subsection{F-OFDM and W-OFDM Signals}

Figure 5a,b depict the PSD of F-OFDM and W-OFDM signals, respectively, at the transmitter and the receiver. The received signals (i) have propagated via an MV-MTL having the features given in Table 1, and (ii) have undergone the superposition of colored Nakagami noise. In both cases, the higher spectral content of the received signal is increased due to the corresponding behavior of the additive noise. The F-OFDM signal plotted in Figure 5a exhibits a main spectral lobe confined within the NB-PLC frequency range $(3-148.5 \mathrm{kHz})$ and a very small $30 \mathrm{~dB}$ transition area. On the other hand, in addition to a main spectral lobe, the W-OFDM signal plotted in Figure 5b exhibits an easily discernible spectral content in the frequency range $220-270 \mathrm{kHz}$. This secondary lobe is the result of the zero padding that is used as input to the IDWT block at the transmitter, which effectively forces W-OFDM to transmit twice the amount of data compared to F-OFDM. It is evident that the transition from the main spectral lobe to the spectral tail is far from being as steep as in F-OFDM. This spectral advantage of F-OFDM is critical, as it results in less inter-carrier interference. The LPF at the receiver is designed to pass only the NB-PLC frequencies, thus suppressing a large part of the quite broader spectral content of the received W-OFDM signal, leading to an increased BER as readily observed from Figure 6. 


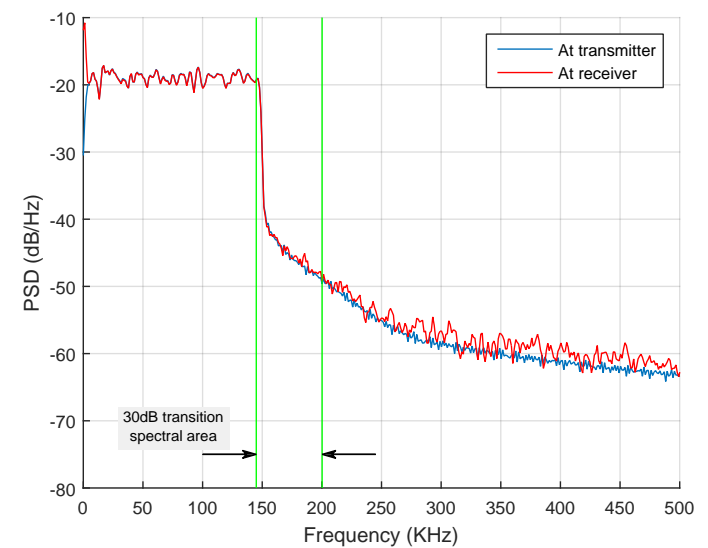

(a)

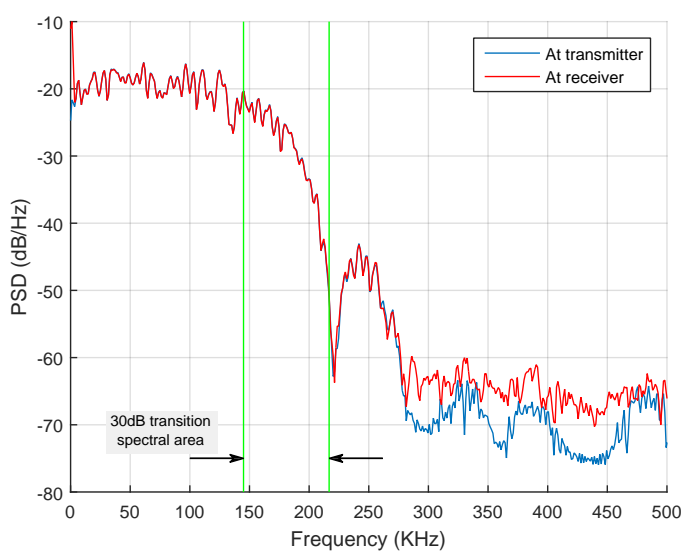

(b)

Figure 5. Spectrum of (a) F-OFDM and (b) W-OFDM signals at the transmitter and the receiver.

\subsection{Maximum Reach of NB-PLC Signals via the MV Grid}

As already mentioned, the primary motivation of this work is to determine the maximum distance between repeaters, say $D_{\max }$, of an NB-PLC system destined for transferring low rate data via non-branched MV power lines. If such a distance is sufficiently large, a smaller number of repeaters will be necessary to transfer low rate SG data from remote nodes on the MV grid-usually in rural and/or remote areas-making such a data transmission technically easier and economically affordable. Here, $D_{\max }$ is determined for various target BER levels at the receiver input employing either F-OFDM or W-OFDM. Figure $6 \mathrm{a}, \mathrm{b}$ show the dependence of BER on $D_{\max }$ when 2-QAM or 4-QAM is employed, respectively. The use of 8-QAM yields BER levels that exceed $10^{-1}$. As a result, the corresponding curves are not presented.

The numerical procedure followed to obtain Figure 6 consists of generating a random bit stream at the transmitter, transforming it into an OFDM band-pass signal, and subsequently transmitting it via the simulated NB-PLC channel. After OFDM demodulation, the decoded bit stream is compared to the transmitted one to determine the BER at the receiver output as a function of the input SNR and end-to-end PLC distance.

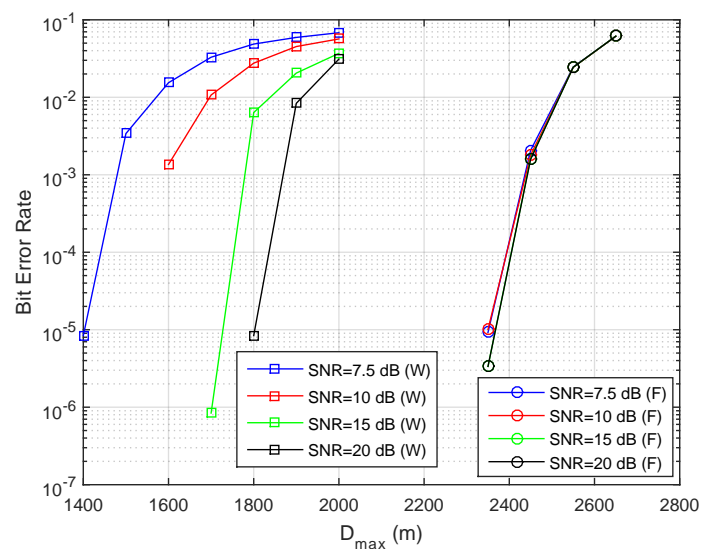

(a)

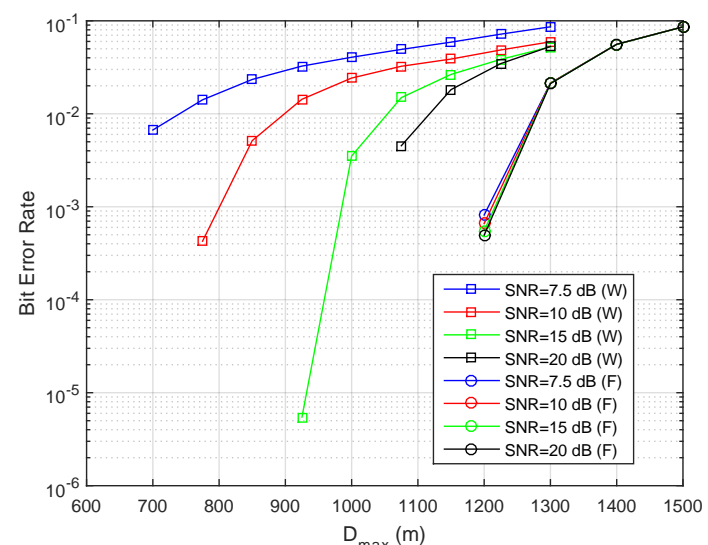

(b)

Figure 6. $D_{\max }$ for various input SNR levels using (a) 2-QAM and (b) 4-QAM (F = F-OFDM, $\mathrm{W}=\mathrm{W}-\mathrm{OFDM})$. 
Figure 6 can be used to determine $D_{\max }$, given the target BER at the receiver which is determined by the specifications of a particular SG service. As switching and monitoring are critical network operations that are not subjected to stringent requirements neither with regard to BER nor to latency, the results of Figure 6 reveal that NB-PLC can be employed to transfer the relevant SG data across remote MV grids. It is also evident that F-OFDM is capable of transmitting NB-PLC signals to significantly greater distances than W-OFDM. In fact, for target BER levels in the range $10^{-5}-10^{-3}$, F-OFDM achieves $D_{\max }$ values ranging from $2350 \mathrm{~m}$ up to $2450 \mathrm{~m}$ using 2-QAM or in the range of $1150 \mathrm{~m}$ up to $1200 \mathrm{~m}$ using 4-QAM. The corresponding ranges when W-OFDM is employed are $1400 \mathrm{~m}$ (for input SNR $=7.5 \mathrm{~dB}$, target $\mathrm{BER}=10^{-5}$ ) up to $1850 \mathrm{~m}$ (for input SNR $=20 \mathrm{~dB}$, target $\mathrm{BER}=10^{-3}$ ) and $600 \mathrm{~m}$ (input $\mathrm{SNR}=7.5 \mathrm{~dB}$, target $\mathrm{BER}=10^{-5}$ ) up to $1000 \mathrm{~m}$ (input $\mathrm{SNR}=20 \mathrm{~dB}$, target $\mathrm{BER}=10^{-3}$ ), respectively. F-OFDM offers a clear transmission range advantage over W-OFDM of at least $500 \mathrm{~m}$ (using 2-QAM) or of at least $150 \mathrm{~m}$ (using 4-QAM). It should be noted that, to obtain the BER results plotted in Figure 6, no use of forward error correction (FEC) has been made. Enhancing NB-PLC transmission with FEC encoding will result in larger $D_{\max }$ values for both F-OFDM and W-OFDM.

Furthermore, as shown by the very small dispersion of the BER curves plotted for various input SNR levels, Figure 6 reveals that the BER performance of F-OFDM when employed for NB-PLC transmission over MV power lines is primarily affected by the distance rather than the noise levels. This is not the case when W-OFDM is employed, as the relevant BER curves are significantly differentiated when the input SNR varies. The less adverse effect of noise on F-OFDM signals compared to $\mathrm{W}-\mathrm{OFDM}$ ones is attributed to the enhanced spectral confinement of the former signals in the NB-PLC frequency range. In addition, due to the spectral confinement of F-OFDM signals, LPF operations at the receiver do not cause significant distortion to the signal.

The results obtained imply that it would be beneficial to extend NB-PLC operation up to $450 \mathrm{kHz}$, as this could offer enhanced SG capabilities over remote MV grids. Therefore, European authorities should consider either licensing or open use of the $148.5-450 \mathrm{kHz}$ spectrum for NB-PLC. In addition, the results obtained may prove very useful for the design of NB-PLC systems over the MV grid. As to $D_{\max }$, which is a crucial parameter, the simulation results show that NB-PLC signals are capable of transmission over greater distances compared to BB-PLC systems, where a repeater is necessary practically every $500-700 \mathrm{~m} \mathrm{[6].}$

Finally, the simulation results are in line with previous experimental data on NB-PLC systems. Successful NB-PLC transmission has been demonstrated over medium voltage power lines that are $1.05 \mathrm{~km}$ and $1.4 \mathrm{~km}$ long [20,21]. The simulation results presented here suggest that those lengths can be extended to even longer distances.

\section{Conclusions}

A parameter drastically affecting the design of PLC systems, as it is directly related to the cost of SG installations, is the distance between repeaters on the same power line. Here, the transmission of NB-PLC signals via non-branched MV power lines is investigated, examining two variants of OFDM, namely filtered-OFDM and wavelet-OFDM. The maximum distance between repeaters is determined, taking into account critical characteristics such as the transfer function of the MTL, the additive noise existing in MV lines in the NB-PLC frequency range and the specific features of the two modulation schemes considered.

The simulation results have shown that when employing either F-OFDM or W-OFDM, the NB-PLC transmission option outperforms BB-PLC, being capable of supporting low rate SG data transmission via MV power lines to distances exceeding $2 \mathrm{~km}$, and that F-OFDM is superior to W-OFDM by $150-500 \mathrm{~m}$, depending on the modulation order. 
Acknowledgments: The authors would like to acknowledge John Dickinson for the helpful discussions.

Author Contributions: Nikolaos Chiotellis performed the simulations presented in the main body of this work and prepared the manuscript. Panayotis Cottis provided guidance with the simulations and edited the manuscript.

Conflicts of Interest: The authors declare no conflict of interest.

\section{References}

1. Fang, X.; Misra, S.; Xue, G.; Yang, D. Smart grid-The new and improved power grid: A survey. IEEE Commun. Surv. Tutor. 2012, 14, 944-980.

2. Livieratos, S.; Vogiatzaki, V.E.; Cottis, P.G. A generic framework for the evaluation of the benefits expected from the smart grid. Energies 2013, 6, 988-1008.

3. Berger, L.T.; Schwager, A.; Escudero-Garzás, J.J. Power line communications for smart grid applications. J. Electr. Comput. Eng. 2013, 2013, doi:10.1155/2013/712376.

4. Lazaropoulos, A.G.; Cottis, P.G. Capacity of overhead medium voltage power line communication channels. IEEE Trans. Power Deliv. 2010, 25, 723-733.

5. IEEE Standards Association. 1901-2010-IEEE Standard for Broadband over Power Line Networks: Medium Access Control and Physical Layer Specifications; IEEE: New York, NY, USA, 2010; pp. 1-1586.

6. Sarafi, A.M.; Tsiropoulos, G.I.; Cottis, P.G. Hybrid wireless-broadband over power lines: A promising broadband solution in rural areas. IEEE Commun. Mag. 2009, 47, 140-147.

7. Ferreira, H.C.; Grové, H.M.; Hooijen, O.; Vinck, A.J. Power Line Communication: An Overview; Wiley Online Library: Hoboken, NJ, USA, 2001.

8. Lazaropoulos, A.G.; Cottis, P.G. Transmission characteristics of overhead medium-voltage power-line communication channels. IEEE Trans. Power Deliv. 2009, 24, 1164-1173.

9. Amore, M.; Sarto, M. A new formulation of lossy ground return parameters for transient analysis of multiconductor dissipative lines. IEEE Trans. Power Deliv. 1997, 12, 303-314.

10. Amirshahi, P.; Kavehrad, M. High-frequency characteristics of overhead multiconductor power lines for broadband communications. IEEE J. Sel. Areas Commun. 2006, 24, 1292-1303.

11. Lee, J.J.; Choi, S.J.; Oh, H.M.; Lee, W.T.; Kim, K.H.; Lee, D.Y. Measurements of the communications environment in medium voltage power distribution lines for wide-band power line communications. In Proceedings of the International Symposium on Power-Line Communications and Its Applications, Zaragoza, Spain, 31 March-2 April 2004; pp. 69-74.

12. Tao, Z.; Xiaoxian, Y.; Baohui, Z.; Jian, C.; Zhi, Y.; Zhihong, T. Research of noise characteristics for 10-kV medium-voltage power lines. IEEE Trans. Power Deliv. 2007, 22, 142-150.

13. Suljanović, N.; Mujčić, A.; Zajc, M.; Tasič, J.F. Corona noise characteristics in high voltage PLC channel. In Proceedings of the 2003 IEEE International Conference on Industrial Technology, Maribor, Slovenia, 10-12 December 2003; Volume 2, pp. 1036-1039.

14. Tao, Z.; Xiaoxian, Y.; Baohui, Z.; Xu, N.H.; Xiaoqun, F.; Changxin, L. Statistical analysis and modeling of noise on 10-kV medium-voltage power lines. IEEE Trans. Power Deliv. 2007, 22, 1433-1439.

15. Farhang, A.; Kakhki, M.M.; Farhang-Boroujeny, B. Wavelet-OFDM versus filtered-OFDM in power line communication systems. In Proceedings of the 2010 5th International Symposium on Telecommunications (IST), Tehran, Iran, 4-6 December 2010; pp. 691-694.

16. Farhang-Boroujeny, B. OFDM versus filter bank multicarrier. IEEE Signal Process. Mag. 2011, 28, 92-112.

17. Sharma, S.; Kumar, S. BER performance evaluation of FFT-OFDM and DWT-OFDM. Int. J. Netw. Mob. Technol. 2011, 2, 110-116.

18. Koga, H.; Kodama, N.; Konishi, T.; Gondo, T. High-speed power line communication system based on wavelet OFDM. In Proceedings of the IEEJ Transactions Electronics, Information and Systems Society National Conference, Kyoto, Japan, 26-28 March 2003; pp. 226-231.

19. Abdullah, K.; Hussain, Z.M. Studies on DWT-OFDM and FFT-OFDM systems. In Proceedings of the IEEE International Conference on Communication, Computer and Power, Muscat, Oman, 15-18 February 2009; pp. 15-18. 
20. Artale, G.; Cataliotti, A.; Cosentino, V.; Di Cara, D.; Fiorelli, R.; Russotto, P.; Tine, G. Medium voltage smart grid: Experimental analysis of secondary substation narrow band power line communication. IEEE Trans. Instrum. Meas. 2013, 62, 2391-2398.

21. Cataliotti, A.; Cara, D.D.; Fiorelli, R.; Tinè, G. Power-line communication in medium-voltage system: Simulation model and onfield experimental tests. IEEE Trans. Power Deliv. 2012, 27, 62-69.

(C) 2016 by the authors; licensee MDPI, Basel, Switzerland. This article is an open access article distributed under the terms and conditions of the Creative Commons by Attribution (CC-BY) license (http:/ / creativecommons.org/licenses/by/4.0/). 